\title{
Tachyonic field interacting with Scalar (Phantom) Field
}

\author{
Surajit Chattopadhyay ${ }^{1 *}$ and Ujjal Debnath ${ }^{2 \dagger}$ \\ ${ }^{1}$ Department of Computer Application, Pailan College of Management and Technology, Bengal Pailan Park, Kolkata-700 104, India. \\ ${ }^{2}$ Department of Mathematics, Bengal Engineering and Science University, Shibpur, Howrah-711 103, India.
}

(Received on 26 November, 2008)

\begin{abstract}
In this letter, we have considered the universe is filled with the mixture of tachyonic field and scalar or phantom field. If the tachyonic field interacts with scalar or phantom field, the interaction term decays with time and the energy for scalar field is transferred to tachyonic field or the energy for phantom field is transferred to tachyonic field. The tachyonic field and scalar field potentials always decrease, but phantom field potential always increases.
\end{abstract}

Keywords: acceleration, tachyonic field, scalar field, phantom field

Recent measurements of the luminosity-redshift relations observed $[1,2]$ for a number of newly discovered type Ia supernova indicate that at present the universe is expanding in a accelerated manner. This has given rise to a lot of dark energy models [3-6], which are supposed to be the reason behind this present acceleration. This mysterious fluid called dark energy is believed to dominate over the matter content of the Universe by $70 \%$ and to have enough negative pressure as to drive present day acceleration. Most of the dark energy models involve one or more scalar fields with various actions and with or without a scalar field potential [7]. The ratio $w$ between the pressure and the energy density of the dark energy seems to be near of less than $-1,-1.62<w<-0.72$ [8]. Numerous models of dark energy exist. There is much interest now in the tachyon cosmology [9] where the appearance of tachyon is basically motivated by string theory [10]. It has been recently shown by Sen $[11,12]$ that the decay of an unstable D-brane produces pressure-less gas with finite energy density that resembles classical dust. The cosmological effects of the tachyon rolling down to its ground state have been discussed by Gibbons [13]. Rolling tachyon matter associated with unstable D-branes has an interesting equation of state which smoothly interpolates between -1 and 0 i.e., $-1<w<0$. As the Tachyon field rolls down the hill, the universe experiences accelerated expansion and at a particular epoch the scale factor passes through the point of inflection marking the end of inflation [10]. The tachyonic matter might provide an explanation for inflation at the early epochs and could contribute to some new form of cosmological dark matter at late times [14]. Inflation under tachyonic field has also been discussed in ref. $[9,15,16]$. Also the tachyon field has a potential which has an unstable maximum at the origin and decays to almost zero as the field goes to infinity. Depending on various forms of this potential following this asymptotic behaviour a lot of works have been carried out on tachyonic dark energy [6, 17]. Sami et al [18] have discussed the cosmological prospects of rolling tachyon with exponential potential.

The phantom field (with negative kinetic energy) [19] was also proposed as a candidate for dark energy as it admits sufficient negative pressure $(w<-1)$. One remarkable feature of the phantom model is that the universe will end

\footnotetext{
*Electronic address: surajit_2008@yahoo.co.in

${ }^{\dagger}$ Electronic address: ujjaldebnath@yahoo.com, ujjalliucaa.ernet.in
}

with a "big rip" (future singularity). That is, for phantom dominated universe, its total lifetime is finite. Before the death of the universe, the phantom dark energy will rip apart all bound structures like the Milky Way, solar system, Earth and ultimately the molecules, atoms, nuclei and nucleons of which we are composed.

To obtain a suitable evolution of the Universe an interaction is often assumed such that the decay rate should be proportional to the present value of the Hubble parameter for good fit to the expansion history of the Universe as determined by the Supernovae and CMB data [20]. These kind of models describe an energy flow between the components so that no components are conserved separately. There are several work on the interaction between dark energy (tachyon or phantom) and dark matter [21], where phenomenologically introduced different forms of interaction term.

Here, we consider a model which comprises of a two component mixture. Here we are interested in how such an interaction between the tachyon and scalar or phantom dark energy affects the evolution and total lifetime of the universe. We consider an energy flow between them by introducing an interaction term which is proportional to the product of the Hubble parameter and the density of the tachyonic field.

The metric of a spatially flat isotropic and homogeneous Universe in FRW model is

$$
d s^{2}=d t^{2}-a^{2}(t)\left[d r^{2}+r^{2}\left(d \theta^{2}+\sin ^{2} \theta d \phi^{2}\right)\right]
$$

where $a(t)$ is the scale factor.

The Einstein field equations are (choosing $8 \pi G=c=1$ )

$$
3 H^{2}=\rho_{t o t}
$$

and

$$
6\left(\dot{H}+H^{2}\right)=-\left(\rho_{t o t}+3 p_{t o t}\right)
$$

where, $\rho_{t o t}$ and $p_{t o t}$ are the total energy density and the pressure of the Universe and $H=\frac{\dot{a}}{a}$ is the Hubble parameter.

The energy conservation equation is

$$
\dot{\rho}_{t o t}+3 H\left(\rho_{t o t}+p_{t o t}\right)=0
$$

Now we consider a two fluid model consisting of tachyonic field and scalar field (or phantom field). Hence the total energy 
density and pressure are respectively given by

$$
\rho_{t o t}=\rho_{1}+\rho_{2}
$$

and

$$
p_{\text {tot }}=p_{1}+p_{2}
$$

The energy density $\rho_{1}$ and pressure $p_{1}$ for tachyonic field $\phi_{1}$ with potential $V_{1}\left(\phi_{1}\right)$ are respectively given by

$$
\rho_{1}=\frac{V_{1}\left(\phi_{1}\right)}{\sqrt{1-\dot{\phi}_{1}^{2}}}
$$

and

$$
p_{1}=-V_{1}\left(\phi_{1}\right) \sqrt{1-\dot{\phi}_{1}^{2}}
$$

The energy density $\rho_{2}$ and pressure $p_{2}$ for scalar field (or phantom field) $\phi_{2}$ with potential $V_{2}\left(\phi_{2}\right)$ are respectively given by

$$
\rho_{2}=\frac{\varepsilon}{2} \dot{\phi}_{2}^{2}+V_{2}\left(\phi_{2}\right)
$$

and

$$
p_{2}=\frac{\varepsilon}{2} \dot{\phi}_{2}^{2}-V_{2}\left(\phi_{2}\right)
$$

where, $\varepsilon=1$ for scalar field and $\varepsilon=-1$ for phantom field.

Therefore, the conservation equation reduces to

$$
\dot{\rho}_{1}+3 H\left(\rho_{1}+p_{1}\right)=-Q
$$

and

$$
\dot{\rho}_{2}+3 H\left(\rho_{2}+p_{2}\right)=Q
$$

where, $Q$ is the interaction term. For getting convenience while integrating equation (11), we have chosen $Q=3 \delta H \rho_{1}$ where $\delta$ is the interaction parameter.

Now eq.(11) reduces to the form

$$
\frac{\dot{V}_{1}}{V_{1}}+\frac{\dot{\phi}_{1} \ddot{\phi}_{1}}{1-\dot{\phi}_{1}^{2}}+3 H\left(\delta+\dot{\phi}_{1}^{2}\right)=0
$$

Here $V_{1}$ is a function of $\phi_{1}$ which is a function of time $t$. Naturally, $\dot{\phi}_{1}$ will be a function of time $t$ and hence it is possible to choose $V_{1}$ as a function of $\dot{\phi}_{1}$. Now, in order to solve the equation (13), we take a simple form of $V_{1}=\left(1-\dot{\phi}_{1}^{2}\right)^{-m},(m>0)$ [22], so that the solution of $\dot{\phi}_{1}$ becomes

$$
\dot{\phi}_{1}^{2}=\left[-\delta+\left(\frac{c}{a^{3}}\right)^{\frac{2(1+\delta)}{1+2 m}}\right]\left[1+\left(\frac{c}{a^{3}}\right)^{\frac{2(1+\delta)}{1+2 m}}\right]^{-1}
$$

where $c$ is an integration constant. The potential $V_{1}$ of the tachyonic field $\phi_{1}$ can be written as

$$
V_{1}=\left[1+\left(\frac{c}{a^{3}}\right)^{\frac{2(1+\delta)}{1+2 m}}\right]^{m}(1+\delta)^{-m}
$$

So from equations (2) and (3), we have

$$
\begin{aligned}
& \dot{\phi}_{2}^{2}=-\frac{2 \dot{H}}{\varepsilon}+\frac{1}{\varepsilon}\left[1+\left(\frac{c}{a^{3}}\right)^{\frac{2(1+\delta)}{1+2 m}}\right]^{m-\frac{1}{2}} \\
& \times\left[\delta-\left(\frac{c}{a^{3}}\right)^{\frac{2(1+\delta)}{1+2 m}}\right](1+\delta)^{-m-\frac{1}{2}}
\end{aligned}
$$

and

$$
\begin{aligned}
& V_{2}=\dot{H}+3 H^{2}-\frac{1}{2}\left[1+\left(\frac{c}{a^{3}}\right)^{\frac{2(1+\delta)}{1+2 m}}\right]^{m-\frac{1}{2}} \\
& \times\left[2+\delta+\left(\frac{c}{a^{3}}\right)^{\frac{2(1+\delta)}{1+2 m}}\right](1+\delta)^{-m-\frac{1}{2}}
\end{aligned}
$$

Now eq.(12) can be re-written as

$$
\dot{V}_{2}+\varepsilon \dot{\phi}_{2} \ddot{\phi}_{2}+3 H\left(\varepsilon \dot{\phi}_{2}^{2}-\delta \rho_{1}\right)=0
$$

Now putting the values of $\dot{\phi}_{2}$ and $V_{2}$, the eq.(18) is automatically satisfied.

Now for simplicity, let us consider $V_{2}=n \dot{\phi}_{2}^{2}$, so from the above equation (17) we have

$$
\begin{aligned}
& \dot{\phi}_{2}^{2}=c_{1}^{2} a^{-\frac{6 \varepsilon}{2 n+\varepsilon}}+\frac{6 \delta}{2 n+\varepsilon}(1+\delta)^{-m-\frac{1}{2}} a^{-\frac{6 \varepsilon}{2 n+\varepsilon}} \\
& \times \int a^{\frac{6 \varepsilon}{2 n+\varepsilon}-1}\left[1+\left(\frac{c}{a^{3}}\right)^{\frac{2(1+\delta)}{1+2 m}}\right]^{m+\frac{1}{2}} d a \\
& =c_{1}^{2} a^{-\frac{6 \varepsilon}{2 n+\varepsilon}}-\delta(1+\delta)^{-m-\frac{1}{2}}{ }_{2} F_{1}\left[\frac{1+2 m}{(1+\delta)(2 n+\varepsilon)},\right. \\
& \left.-m-\frac{1}{2}, 1+\frac{1+2 m}{(1+\delta)(2 n+\varepsilon)},-\left(\frac{c}{a^{3}}\right)^{\frac{2(1+\delta)}{1+2 m}}\right]
\end{aligned}
$$

and

$$
\begin{aligned}
& V_{2}=n c_{1}^{2} a^{-\frac{6 \varepsilon}{2 n+\varepsilon}}-n \delta(1+\delta)^{-m-\frac{1}{2}}{ }_{2} F_{1}\left[\frac{1+2 m}{(1+\delta)(2 n+\varepsilon)}\right. \\
& \left.-m-\frac{1}{2}, 1+\frac{1+2 m}{(1+\delta)(2 n+\varepsilon)},-\left(\frac{c}{a^{3}}\right)^{\frac{2(1+\delta)}{1+2 m}}\right]
\end{aligned}
$$

Figures 1 - 6 are drawn for scalar field model with $\delta=-0.05$ and figures $7-12$ are drawn for phantom model with $\delta=-0.05$. Figs. $1-4$ show the variations $\rho_{1}, \rho_{2}, H, Q$ with redshift $z$ and figs. 5, 6 show the variations of $V_{1}$ with $\phi_{1}, V_{2}$ with $\phi_{2}$ respectively for scalar field model. Figs. 7 - 10 show the variations $\rho_{1}, \rho_{2}, H, Q$ with redshift $z$ and figs. 11 , 12 show the variations of $V_{1}$ with $\phi_{1}, V_{2}$ with $\phi_{2}$ respectively for phantom model. For scalar field model $\rho_{1}, \rho_{2}, H, Q$ decrease with decreasing $z$ but for phantom model $\rho_{1}, \rho_{2}, H$ decrease first and then increase with decreasing $z$ and $Q$ decreases with decreasing $z$. The tachyonic field and scalar field potentials always decrease, but phantom field potential always increases. For scalar field model $(\varepsilon=+1)$ and phantom phantom field model $(\varepsilon=-1), \delta$ may be negative due to positivity of $\dot{\phi}_{2}^{2}$. So from equations (11) and (12), it may be concluded that the energy for scalar field will be transferred to tachyonic field the energy for phantom field will be transferred to tachyonic field. In both the cases, the interaction term 


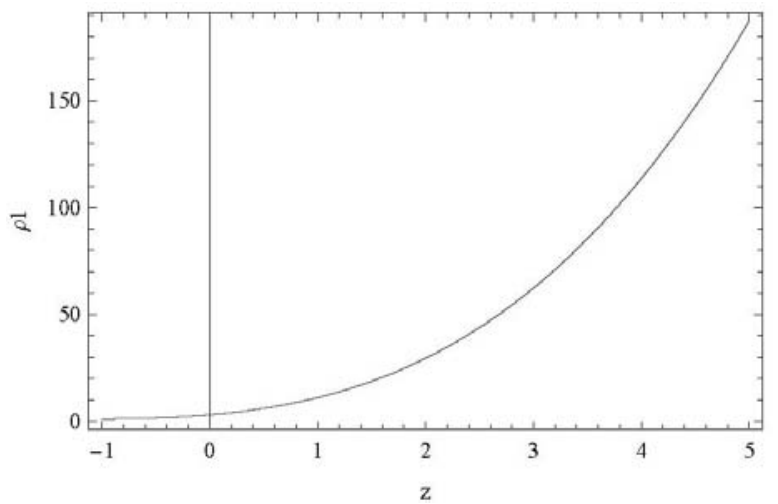

Fig.1

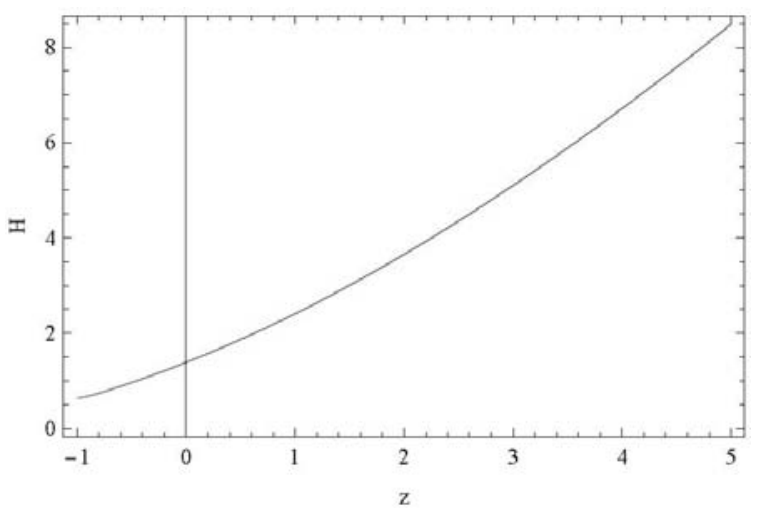

Fig.3

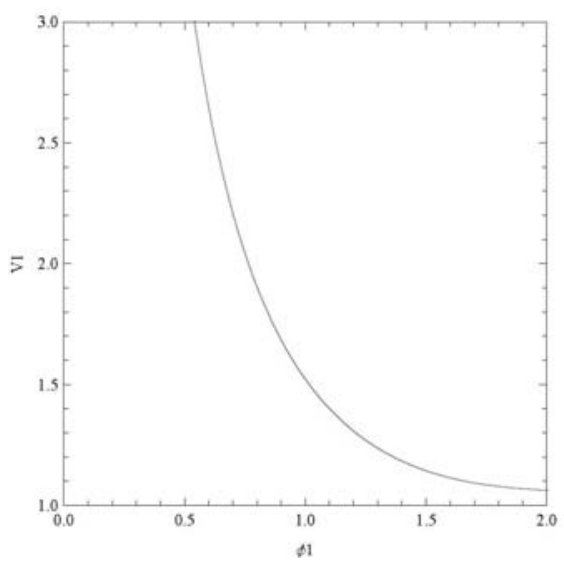

Fig.5

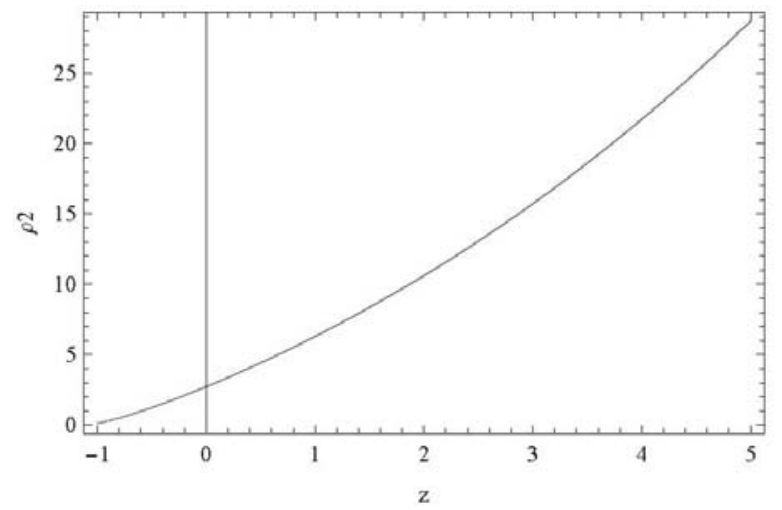

Fig.2

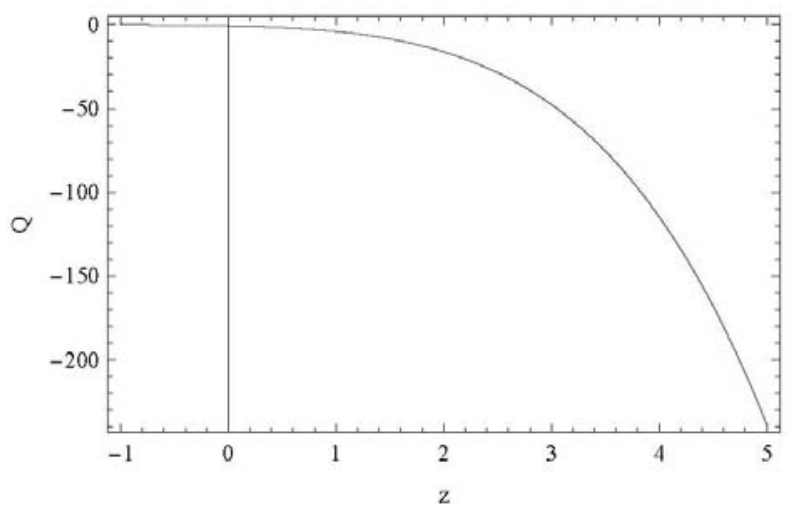

Fig.4

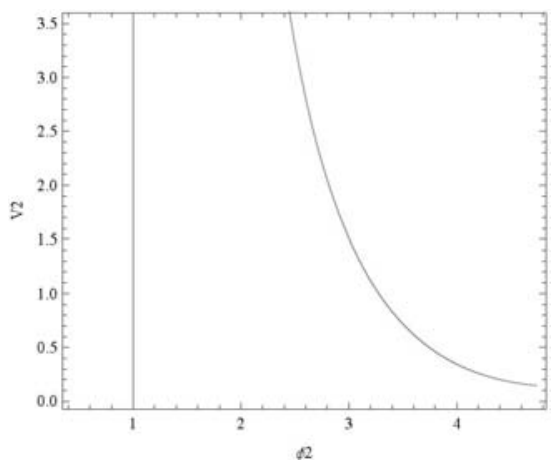

Fig.6

Figs. 1, 2, 3 and 4 show variations of $\rho_{1}, \rho_{2}, H$ and $q$ respectively with redshift $z$ and figs. 5, 6 show variations of $V_{1}$ with $\phi_{1}, V_{2}$ with $\phi_{2}$ respectively for $\varepsilon=+1, n=2, \delta=-0.05, m=c=c 1=1$.

is decays with time. Figures 13 - 16 are generated taking $\delta=0$. Comparing figures $5,6,11$ and 12 with figures 13 , 14, 15 and 16, we have seen that interaction does not have any significant impact on the variations of potentials against fields. This may be due to the fact that interaction is very small. 


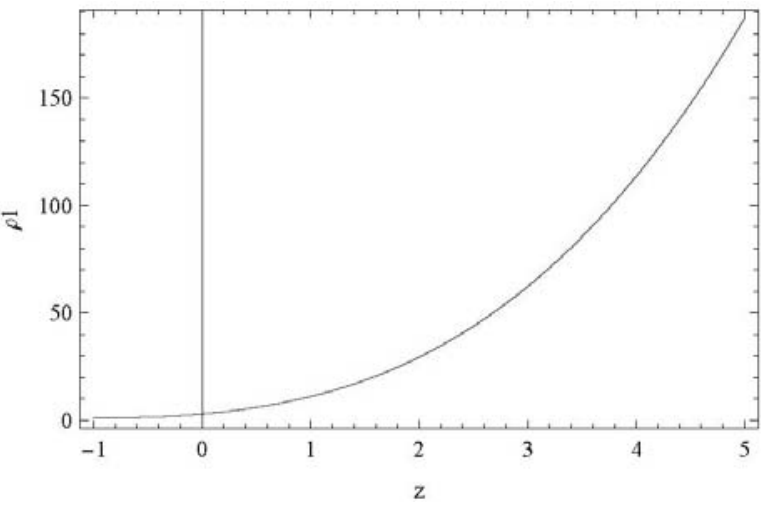

Fig.7

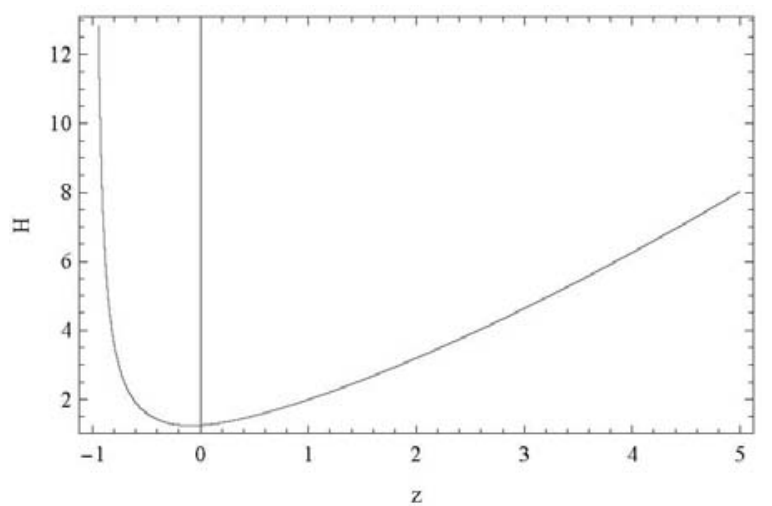

Fig.9

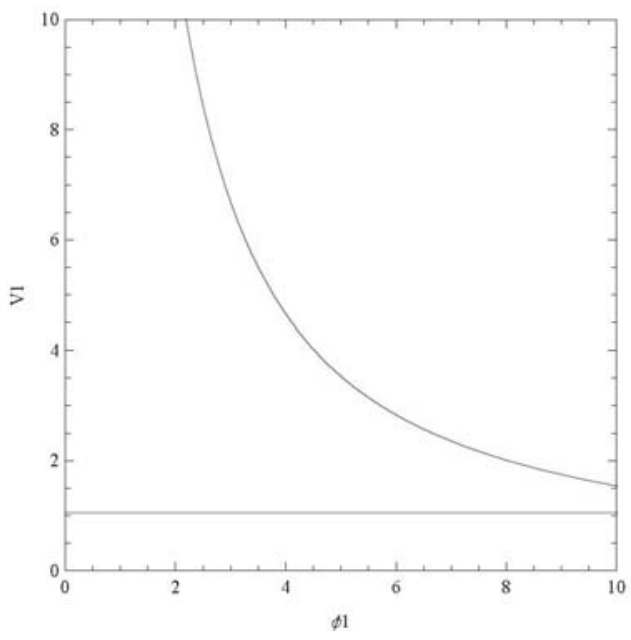

Fig. 11

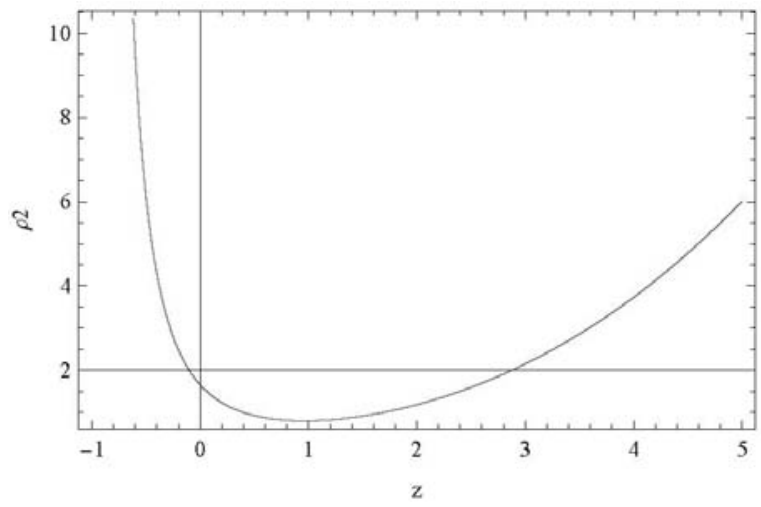

Fig.8

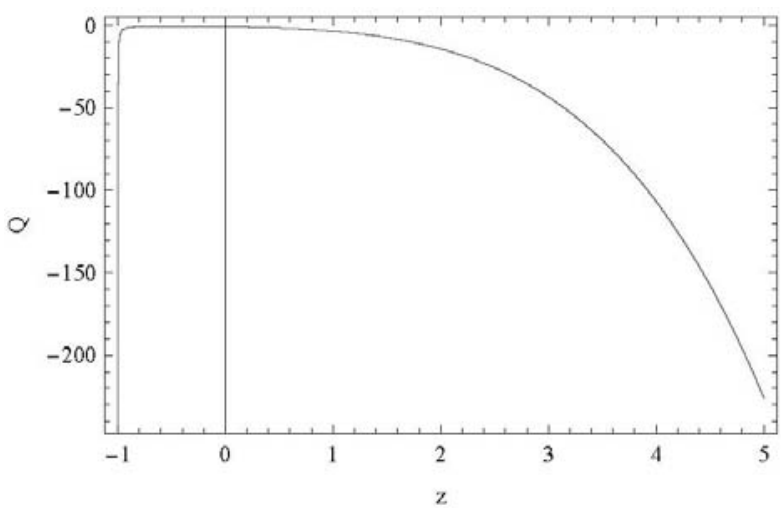

Fig.10

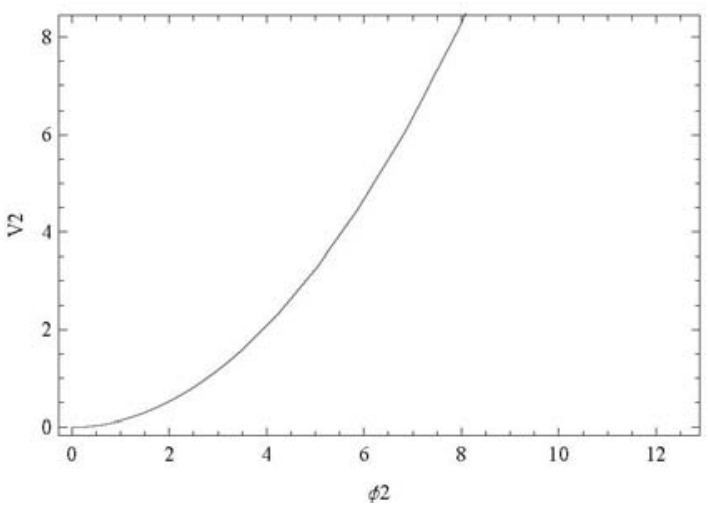

Fig. 12

Figs. 7, 8, 9 and 10 show variations of $\rho_{1}, \rho_{2}, H$ and $q$ respectively with redshift $z$ and figs. 11,12 show variations of $V_{1}$ with $\phi_{1}, V_{2}$ with $\phi_{2}$ respectively for $\varepsilon=-1, n=2, \delta=-0.05, m=c=c 1=1$. 


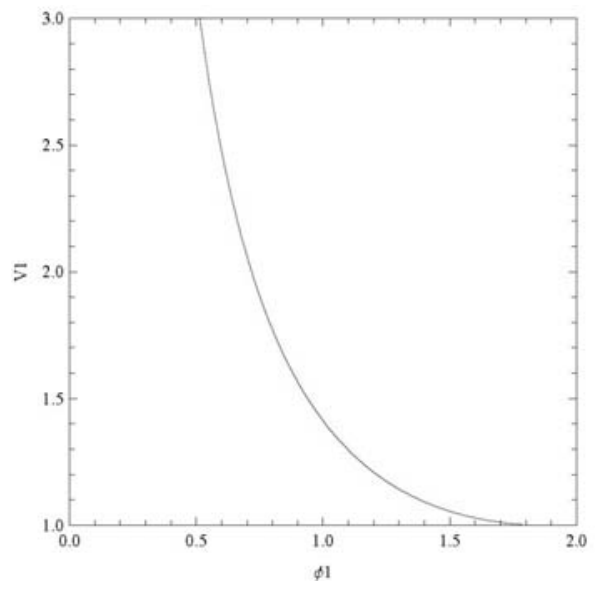

Fig.13

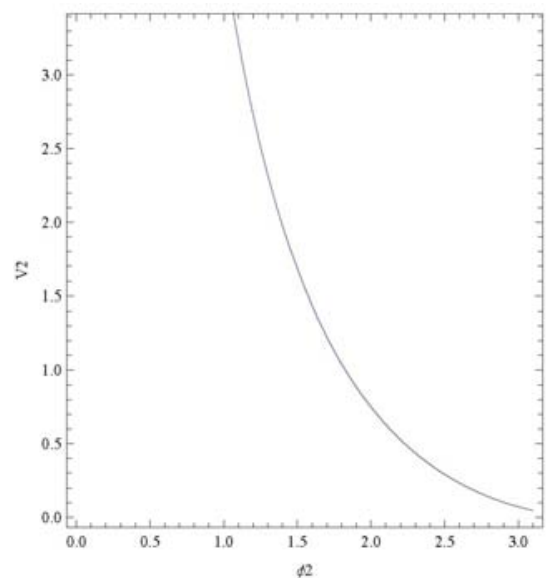

Fig. 14

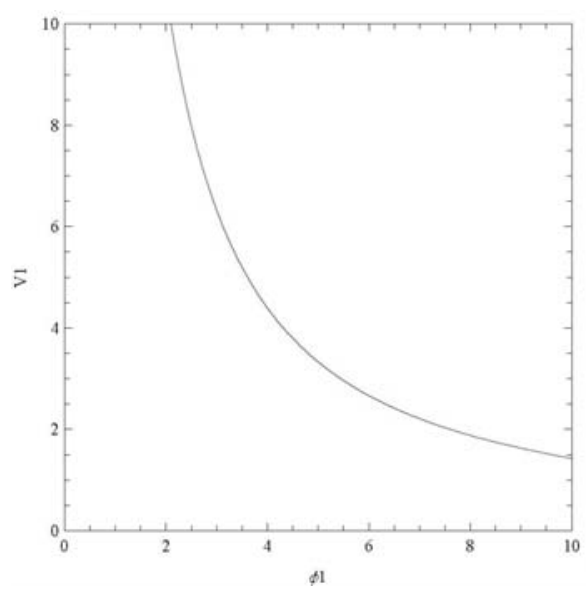

Fig.15

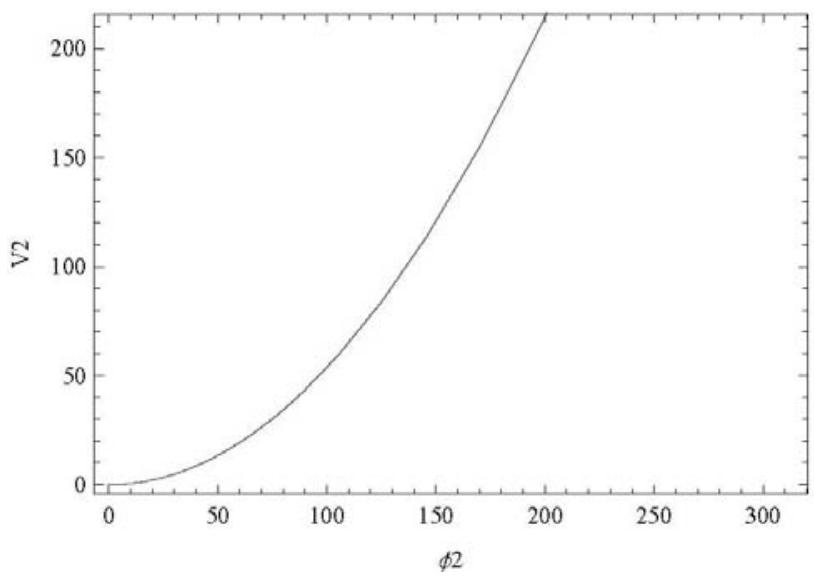

Fig.16

Figs. 13,14 show the variations of $V_{1}$ with $\phi_{1}, V_{2}$ with $\phi_{2}$ respectively for $\varepsilon=+1, n=2, \delta=0, m=c=c 1=1$ and Figs. 15,16 show the variations of $V_{1}$ with $\phi_{1}, V_{2}$ with $\phi_{2}$ respectively for $\varepsilon=-1, n=2, \delta=0, m=c=c 1=1$.

\section{Acknowledgement}

The authors wish to acknowledge the warm hospitality provided by IUCAA, Pune, India, where part of the work was carried out. One of the authors (UD) is thankful to UGC, Govt. of India for providing research project grant (No. 32$157 / 2006($ SR)).
[1] N. A. Bachall, J. P. Ostriker, S. Perlmutter and P. J. Steinhardt, Science 2841481 (1999).

[2] S. J. Perlmutter et al, Astrophys. J. 517565 (1999).

[3] V. Sahni and A. A. Starobinsky, Int. J. Mod. Phys. A 9373 (2000).

[4] P. J. E. Peebles and B. Ratra, Rev. Mod. Phys. 75559 (2003).

[5] T. Padmanabhan, Phys. Rept. 380235 (2003).

[6] E. J. Copeland, M. Sami, S. Tsujikawa, Int. J. Mod. Phys. D 15 1753 (2006).

[7] I. Maor and R. Brustein, Phys. Rev. D 67103508 (2003); V. H. Cardenas and S. D. Campo, Phys. Rev. D 69083508 (2004); P.G. Ferreira and M. Joyce, Phys. Rev. D 58023503 (1998).

[8] A. Melchiorri, L. Mersini, C. J. Odmann and M. Trodden, Phys. Rev. D 68043509 (2003).
[9] A. Feinstein, Phys. Rev. D 66063511 (2002).

[10] M. Sami, Mod. Phys. Lett. A 18691 (2003).

[11] A. Sen, JHEP 0204048 (2002).

[12] A. Sen, JHEP 0207065 (2002).

[13] G. W. Gibbons, Phys. Lett. B 5371 (2002).

[14] M. Sami, P. Chingangbam and T. Qureshi, Phys. Rev. D 66 043530 (2002).

[15] M. Fairbairn and M.H.G. Tytgat, Phys. Lett. B 5461 (2002).

[16] T. Padmanabhan, Phys. Rev. D 66021301 (2002).

[17] J. S. Bagla, H. K. Jassal and T. Padmanabhan, Phys. Rev. D 67 063504 (2003); E. J. Copeland, M. R. Garousi, M. Sami and S. Tsujikawa, Phys. Rev D 71043003 (2005); G. Calcagni and A. R. Liddle, Phys. Rev. D 74043528 (2006).

[18] M. Sami, P. Chingangbam and T. Qureshi, Pramana 62765 
(2004).

[19] L. Parker and A. Raval, Phys. Rev. D 60063512 (1999); D. Polarski and A. A. Starobinsky, Phys. Rev. Lett. 852236 (2000); R. R. Caldwell, Phys. Lett. B 54523 (2002).

[20] M. S. Berger, H. Shojaei, Phys. Rev. D 74043530 (2006).

[21] R. Herrera, D. Pavon, W. Zimdahl, Gen. Rel. Grav. 362161
(2004); R. -G. Cai and A. Wang, JCAP 0503002 (2005); Z.K. Guo, R.-G. Cai and Y.-Z. Zhang, JCAP 0505002 (2005); T. Gonzalez and I. Quiros, $g r-q c / 0707.2089$.

[22] S. Chattopadhyay, U. Debnath and G. Chattopadhyay, Astrophys. Space Sci. 31441 (2008). 